\title{
LASIK and PRK in hyperopic astigmatic eyes: is early retreatment advisable?
}

\author{
This article was published in the following Dove Press journal: \\ Clinical Ophthalmology \\ 31 March 2016 \\ Number of times this article has been viewed
}

\author{
Andreas Frings 1 \\ Gisbert Richard ${ }^{1,2}$ \\ Johannes Steinberg ${ }^{1,3,4}$ \\ Vasyl Druchkiv ${ }^{1,4}$ \\ Stephan Johannes Linke \\ Toam Katz ${ }^{1,4}$ \\ 'Department of Ophthalmology, \\ University Medical Centre Hamburg- \\ Eppendorf, ${ }^{2} \mathrm{Ophthalmologikum} \mathrm{an}$ \\ der Alster, ${ }^{3}$ zentrumsehstärke, ${ }^{4} \mathrm{CARE}$ \\ Vision Germany GmbH, Hamburg, \\ Germany
}

Purpose: To analyze the refractive and keratometric stability in hyperopic astigmatic laser in situ keratomileusis (LASIK) or photorefractive keratectomy (PRK) during the first 6 months after surgery.

Patients and methods: This retrospective cross-sectional study included 97 hyperopic eyes; 55 were treated with LASIK and 42 with PRK. Excimer ablation for all eyes was performed using the ALLEGRETTO excimer laser platform using a mitomycin C for PRK and a mechanical microkeratome for LASIK. Keratometric and refractive data were analyzed during three consecutive follow-up intervals ( 6 weeks, 3 months, and 6 months). The corneal topography was obtained using Scheimpflug topography, and subjective refractions were acquired by expert optometrists according to a standardized protocol.

Results: After 3 months, mean keratometry and spherical equivalent were stable after LASIK, whereas PRK-treated eyes presented statistically significant $(P<0.001)$ regression of hyperopia. In eleven cases, hyperopic regression of $>1 \mathrm{D}$ occurred. The optical zone diameter did not correlate with the development of regression.

Conclusion: After corneal laser refractive surgery, keratometric changes are followed by refractive changes and they occur up to 6 months after LASIK and for at least 6 months after PRK, and therefore, caution should be applied when retreatment is planned during the 1st year after surgery because hyperopic refractive regression can lead to suboptimal visual outcome. Keratometric and refractive stability is earlier achieved after LASIK, and therefore, retreatment may be independent of late regression.

Keywords: hyperopia, astigmatism, regression, keratometry

\section{Introduction}

The safety and efficacy of laser in situ keratomileusis (LASIK) and photorefractive keratectomy (PRK) in correcting hyperopic astigmatism have been reported. ${ }^{1}$ However, the efficacy of any corneal refractive surgery relies on refractive and keratometric stability.

Refractive hyperopic regression is common after hyperopic treatment and, therefore, limits the hyperopic correction range to 3-4 D of hyperopia compared with the more than double dioptric range for myopic correction. ${ }^{1-4}$ In our experience, refractive hyperopic regression of $>0.25 \mathrm{D}$ after 3 months caused by hyperopic refractive shift associated with changes in the crystalline lens (due to age) represents a considerable refractive problem, demanding a retreatment in a significant proportion of cases. Since refractive patients often request the earliest possible retreatment, this study analyses the refractive and keratometric stability during the first 6 months after LASIK or PRK in hyperopic astigmatic eyes.
Correspondence: Andreas Frings

Department of Ophthalmology, University Medical Centre Hamburg-

Eppendorf, Martinistraße 52, 20246

Hamburg, Germany

Tel $+494074 I 0$ I8246

Fax +49 40741054906

Email andi.frings@gmail.com 


\section{Materials and methods}

\section{Patients and examinations}

This retrospective study included 97 eyes from 97 hyperopic patients consecutively treated between August 1, 2013, and January 5, 2014, and was based on the Hamburg Refractive Database (data retrieved from Care Vision Refractive Centers in Germany). For each patient, one randomly selected eye was analyzed. The inclusion criteria were LASIK and PRK for hyperopia with $>0.5 \mathrm{D}$, no previous refractive surgery, and follow-up (FU) examinations at 6 weeks, 3 months, and 6 months after surgery with full refractive data available.

The written informed consent for retrospective data analysis was obtained from refractive surgery candidates during their recruiting process. The study and consent procedure were approved by the University of Hamburg Ethics Committee (no 2882) and adhered to the tenets of the Declaration of Helsinki.

The hyperopic correction was based on the manifest refraction and presented here as spherical equivalent (SE). Whether a PRK or a LASIK was performed depends on the preoperative pachymetry, topography, and subsequent assumed risk of ectasia as well as on individual risk profile for flap-associated complications. PRK was preferred in patients with hobbies or jobs with a higher risk of eye trauma or in eyes with mild ectasia risk. Moreover, previous studies reported that epithelial remodeling following PRK may be substantially more in comparison to LASIK. ${ }^{5}$ These unavoidable inherent differences in pachymetry and refractions between LASIK and PRK eyes may be statistically disadvantageous but clinically speaking it reflects the actual population of LASIK and PRK patients and is useful for drawing clinical conclusions for these populations.

Manifest spherical and cylindrical refraction, cycloplegic refraction, as well as visual acuity with and without correction were assessed pre- and postoperatively (except cycloplegic refraction). FU included examinations at 1 day (LASIK) to 5 days (PRK) after surgery, and during three consecutive FU examinations ( 6 weeks, 3 months, and 6 months after surgery), and were recorded electronically.

Refractive stability was defined as a maximum of $\pm 0.25 \mathrm{D}$ change in SE or mean keratometry $(\mathrm{Km})$ between 3-month and 6-month examination. The corneal topography was obtained using Scheimpflug topography (Pentacam HR; Oculus, Wetzlar, Germany). All refractions were acquired by expert optometrists using the same refractometers, visual acuity tables, and documentation protocol. Each patient was examined pre- and postoperatively by the same optometrist. Examinations were carried out according to a standardized protocol. To minimize the potential bias, latent hyperopia eyes with preoperative cycloplegic refraction of $>1 \mathrm{D}$ difference to manifest refraction were excluded from this study.

\section{Laser treatment}

Excimer ablation for all eyes was performed using the ALLEGRETTO excimer laser platform (Eye-Q $400 \mathrm{~Hz}$; WaveLight $\mathrm{GmbH}$, Erlangen, Germany) under constant eye tracking using a wavefront-optimized ablation protocol. The surgical procedures using mitomycin $\mathrm{C}$ for PRK and a mechanical microkeratome (SBK $90 \mu \mathrm{m}$; Moria SA, Antony, France) for LASIK have been described previously. ${ }^{6,7}$

The ablation was centered on the visual axis (first Purkinje image), which is required in hyperopic eyes with relatively large-angle kappa. The patient was asked to focus on a target light offered by the excimer platform. The first Purkinje image of this light and its relation to the pupil center were documented, and the eye tracker used this reference point as ablation center ("off-set"). All eyes were treated with an optical zone (OZ) of either $6 \mathrm{~mm}(\mathrm{n}=16), 6.5 \mathrm{~mm}(\mathrm{n}=65)$, or $7 \mathrm{~mm}(\mathrm{n}=16)$ according to scotopic pupil size. The betweengroup difference was not statistically significant (tested with chi-square, $P=0.180$ ).

All three refractive surgeons were experienced consultants and followed a standard protocol of indications, and the pre-, intra-, and postoperative treatment plan was written and implemented by the last author in all three refractive centers involved in this study.

Pre- and postoperative $\mathrm{Km}$ readings were measured in the central $3 \mathrm{~mm}$ as simulated $\mathrm{K}$ ( $\operatorname{simK}$ ) in millimeter radius and converted to D using the air to stroma refraction index of $1: 1.367$. The central $3 \mathrm{~mm}$ includes the visual axis and reflects the main refractive change in the whole cornea. The difference between the true anterior corneal $\mathrm{Km}$ preoperatively and postoperatively follows precisely the change in manifest refraction and hence is an appropriate parameter for the corneal and refractive development over time. The targeted $\mathrm{Km}$ was calculated using the preoperative $\mathrm{Km}$ and adding the SE that was used by the laser platform with compensation for the vertex distance of the manifest refraction $(12 \mathrm{~mm})$ to corneal plane $(0 \mathrm{~mm})$. For example, correcting a manifest $\mathrm{SE}$ of $+4.0 \mathrm{D}$ should increase the central $\mathrm{Km}$ by $4 /(1-0.012 \times 4)=4.2 \mathrm{D}$. In an eye with preoperative $\mathrm{Km}$ of $39 \mathrm{D}$ and SE of $+4 \mathrm{D}$, the treatment should result in $\mathrm{Km}$ of $43.2 \mathrm{D}$ and $\mathrm{SE}$ of $0 \mathrm{D}$. The expected change is in $\mathrm{Km}$ of $+4.2 \mathrm{D}$ and in $\mathrm{SE}$ of +4.0 D. We calculated the postoperative actual $\mathrm{Km}$ and $\mathrm{SE}$ at each $\mathrm{FU}$ and analyzed changes between the FU examinations. 


\section{Statistical analysis}

To analyze the differences in biometrical data between LASIK and PRK, either a parametrical independent $t$-test or a nonparametric Mann-Whitney test was used, depending on the normality of the data. To analyze the predictability of the treatment, linear regression was applied. Changes in SE and $\mathrm{Km}$ during FU were evaluated using repeated measure analysis of variance. The differences between achieved SE change and achieved $\mathrm{Km}$ change were tested with a paired $t$-test.

\section{Results}

Table 1 gives the preoperative refractive data for both LASIK and PRK groups and demonstrates that there were no statistically significant differences except for preoperative pachymetry. In 55 eyes, LASIK was performed and another 42 patients were treated with PRK.

The postoperative data originate from the 6-month FU examination. There were no statistically significant differences in any refractive parameter after surgery (Table 1). Figure 1 shows the development of $\mathrm{Km}$ and $\mathrm{SE}$ during FU. In the LASIK group (Figure 1A; Table 2), postoperative changes in $\mathrm{Km}$ and SE were significant $(P<0.001)$ until 3 months. In other words, there was statistically significant change in refractive and keratometric stability $(P<0.001)$ (Table 2).

In the 42 eyes that had PRK, postoperative changes in $\mathrm{Km}$ and refraction were significant $(P<0.001)$ up to 6 months after surgery (Figure 1B, Table 2). Km and SE progressed in terms of hyperopic regression (= undercorrection) (Figure 1C). In eleven cases, refractive regression was $>1$ D (three LASIK and eight PRK eyes), and this difference was statistically significant between LASIK and PRK (chi-square, $P=0.036$ ).

All laser treatments applied an OZ of either $6 \mathrm{~mm}$, $6.5 \mathrm{~mm}$, or $7 \mathrm{~mm}$ based on scotopic pupil size; there was no statistically significant difference in OZ between LASIK and PRK. The OZ diameter did not correlate with keratometric or refractive regression.

\section{Discussion}

This study analyses the refractive and keratometric stability in hyperopic astigmatic LASIK or PRK during the first 6 months after surgery.

Our results show that stability of refraction and $\mathrm{Km}$ was earlier presented in eyes after LASIK; PRK-treated eyes showed statistically significant higher hyperopic regression displayed by changes in postoperative $\mathrm{SE}$ and $\mathrm{Km}$ up to 6 months after surgery. In 11 (11\%) cases, regression of $>1$ D occurred. Among them were three LASIK- $(5.45 \%$ of all LASIK cases) and eight PRK-treated eyes (19.05\% of all PRK cases). As refractive and topographic stability in LASIK is present at the 6-month FU examination, retreatment could be performed earlier than in PRK eyes. After PRK, we do not recommend any retreatment within the first 6 months after surgery due to hyperopic regression and recommend reevaluation of refractive and topographic stability after 1 year. In general, $\mathrm{Km}$ and refraction developed in a parallel manner that implies that regression is $\mathrm{Km}$ driven and not due to latent hyperopia; moreover, it was independent of OZ diameter.

Refractive stability after hyperopic corneal refractive surgery is still controversial. After LASIK, stability has been reported after 1 month, ${ }^{8}$ at 3 months, ${ }^{9}$ at 6 months, ${ }^{10}$ and at 12 months. ${ }^{11}$ Nevertheless, caution should be applied as refractive stability is often not clearly defined or hard to compare between studies. In our opinion, clinically relevant refractive stability should be defined as a maximum of $\pm 0.25 \mathrm{D}$ change in $\mathrm{SE}$ or $\mathrm{Km}$ after at least 3 months.

We are aware of the fact that the hyperopic shaping of the cornea is three-dimensional and includes multiple changes; however, we chose the simK parameter because it is the only single parameter that presents the total corneal refractive power as close as possible and was measured in all $\mathrm{FU}$ examinations and its change corresponds to the change in refraction. ${ }^{12}$

Both PRK and LASIK are effective and safe in the correction of hyperopia. However, PRK tends to lead to initial temporary myopic overshoot, which can be followed by a hyperopic regression over months to years. ${ }^{1}$ The results of our study concur with that finding (Figure 1; Table 2). de Ortueta and Arba Mosquera showed that topography can be used as an objective method to analyze regression after LASIK for hyperopia. ${ }^{2}$ They reported that virtually no topographic regression between 3-month and 36-month FU could be observed; between 12 months and 36 months a regression of $0.001 \mathrm{D} /$ month was calculated and this was not statistically significant. ${ }^{2}$ Others have reported that regression can occur for years; Jaycock et al found that regression could not be explained by age-related changes in refraction that still occurred after 5 years. ${ }^{3}$ By contrast, Kezirian et al, observing a 4.5-year period after surgery, demonstrated that the selection of the 6-month examination as the time point of stability was justified. ${ }^{4}$ This conclusion is supported by our data showing stability in LASIK-treated eyes after 3 months.

Other factors that have been related to the impact of LASIK on spherical aberration include surface smoothing as a result of corneal wound healing and epithelial 


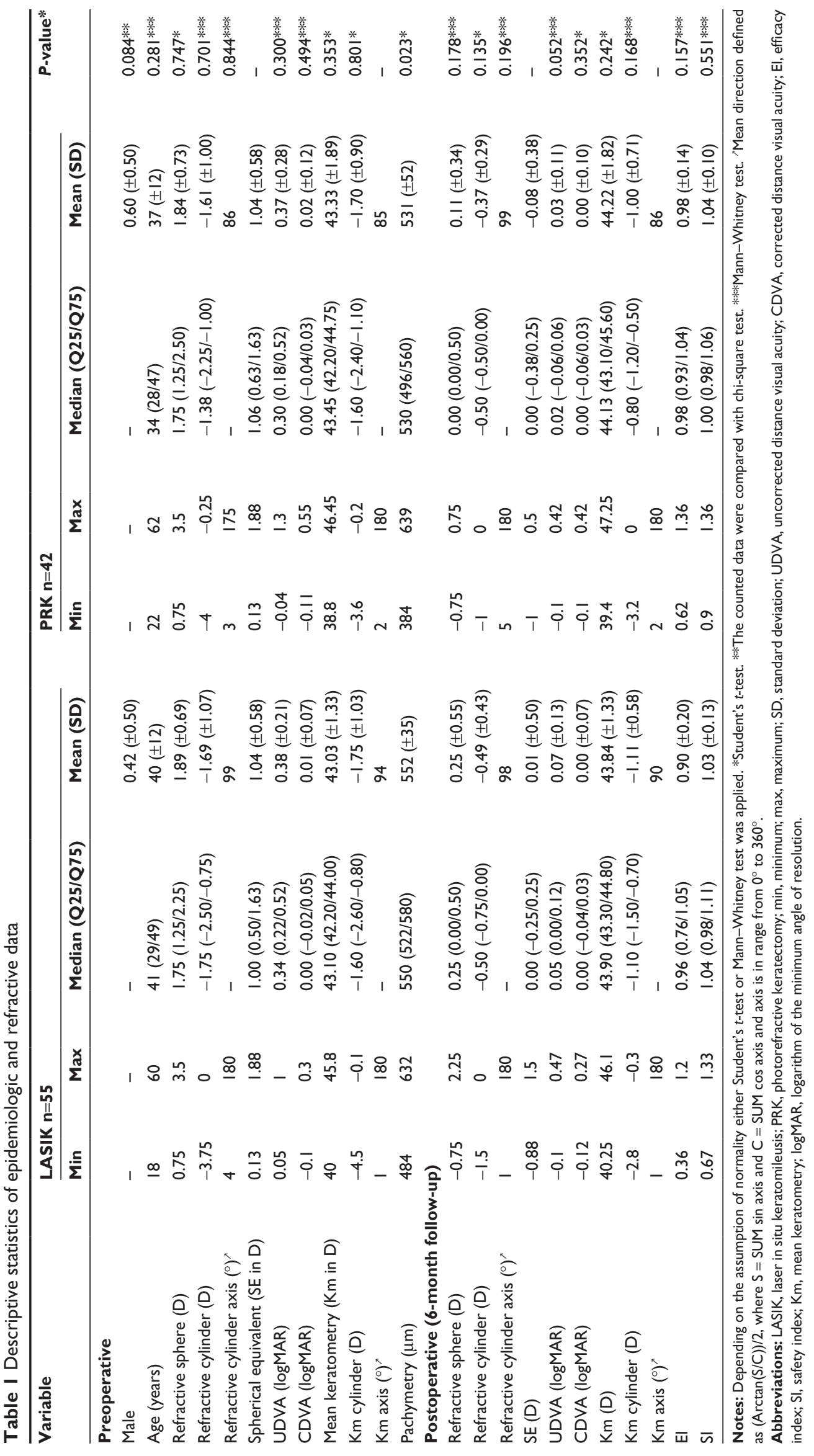



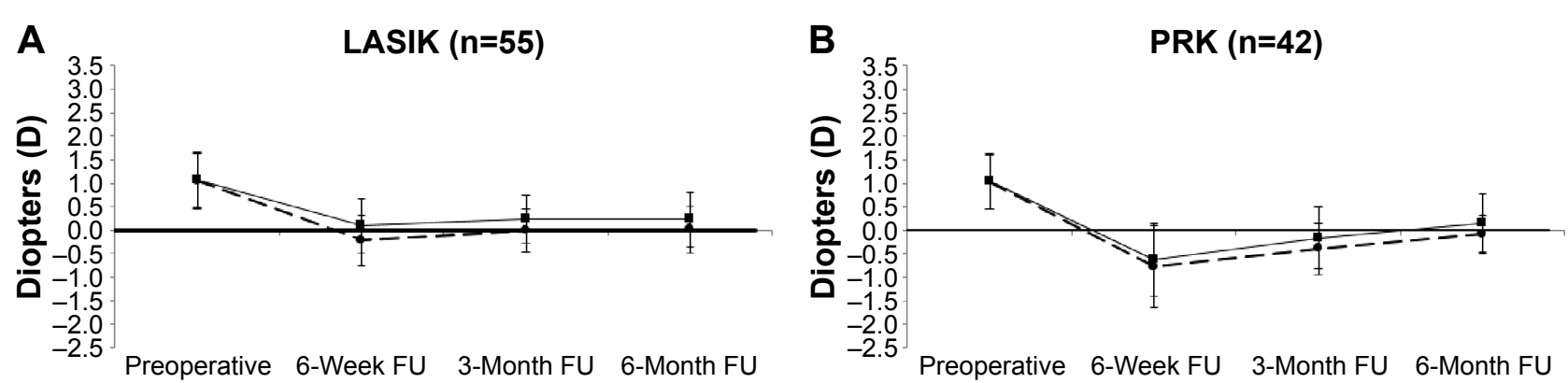

\begin{tabular}{|c|c|c|c|}
\hline & & $\rightarrow$ K-mean $\rightarrow$ SE & \multirow[b]{2}{*}{ PRK } \\
\hline & & LASIK & \\
\hline & & $\overline{\text { Mean (SD) }}$ & $\overline{\text { Mean (SD) }}$ \\
\hline \multirow[t]{2}{*}{$\overline{S E}(D)$} & 6-Week vs 3-Month FU & $0.30(0.31)$ & $0.60(0.55)$ \\
\hline & 3-Month vs 6-Month FU & $0.28(0.25)$ & $0.45(0.31)$ \\
\hline \multirow[t]{2}{*}{$\mathrm{Km}(\mathrm{D})$} & 6-Week vs 3-Month FU & $0.40(0.35)$ & $0.63(0.57)$ \\
\hline & 3-Month vs 6-Month FU & $0.38(0.38)$ & $0.53(0.51)$ \\
\hline
\end{tabular}

Figure I Progression of $\mathrm{Km}$ and SE (in D) within the first 6 months after surgery (6-week, 3-month, and 6-month FU).

Notes: (A) after LASIK, (B) after PRK, and (C) absolute change in $\mathrm{Km}$ and SE.

Abbreviations: Km, mean keratometry; SE, spherical equivalent; FU, follow-up; LASIK, laser in situ keratomileusis; PRK, photorefractive keratectomy, SD; standard deviation.

remodulation, ${ }^{13}$ biomechanical changes, ${ }^{14}$ and variation in the laser ablation depth per pulse across the treatment area in relation to corneal curvature. ${ }^{15}$ This first factor is minimized in our study by using a wavefront-optimized ablation protocol.

In eyes after PRK, regression could be explained by a prolonged epithelial healing process. As previously described, after hyperopic PRK, epithelial remodeling aims to compensate for the ablated corneal tissue. ${ }^{1}$ On the other hand, central epithelial hyperplasia would manifest as refraction overshoots over the first week to month, and after complete healing causes regression after 3-4 months.

Regression has also been strongly associated with the magnitude of hyperopia and some argue that less regression

Table 2 Postoperative changes in $\mathrm{Km}$ and SE in PRK- and LASIKtreated eyes, displayed by follow-up

\begin{tabular}{|c|c|c|}
\hline & After LASIK & After PRK \\
\hline \multicolumn{3}{|c|}{ Combined changes in $\mathrm{Km}$ and SE between consecutive FU } \\
\hline \multicolumn{3}{|l|}{ intervals } \\
\hline 6-Week vs 3-Month FU & $P<0.00 I, \eta^{2}=0.234$ & $P<0.001, \eta^{2 a}=0.333$ \\
\hline 3-Month vs 6-Month FU & $P=0.964, \eta^{2}<0.001$ & $P<0.001, \eta^{2}=0.389$ \\
\hline \multicolumn{3}{|c|}{ Difference between $\mathrm{Km}$ and SE within FU intervals } \\
\hline 6-Week FUb & $P<0.00$ I & $P=0.164$ \\
\hline 3-Month FU & $P=0.00 \mathrm{I}$ & $P=0.037$ \\
\hline 6-Month FU & $P=0.008$ & $P=0.014$ \\
\hline
\end{tabular}


differences between SE and $\mathrm{Km}$ within the intervals.

Abbreviations: $\mathrm{Km}$, mean keratometry; SE, spherical equivalent; PRK, photorefractive keratectomy; LASIK, laser in situ keratomileusis; FU, follow-up. goes along with the use of larger $\mathrm{OZs},{ }^{16}$ it is considerably greater in high hyperopia and can continue up to 12 months. ${ }^{17}$ In our study, there was no statistically significant correlation of $\mathrm{OZ}$ diameter or magnitude of preoperative hyperopia with regression during FU. Moreover, there was no statistically significant difference in OZ between LASIK and PRK groups. Since simK is taken from the central $3 \mathrm{~mm}$, the $\mathrm{OZ}$ should not affect the expected central $\mathrm{Km}$.

In contrast to previous literature, advantages of our study are that we analyzed only one eye per patient who were of relatively young age, we compared two homogeneous groups of LASIK and PRK, and the ablation profiles took into account intraoperative off-set. On the other hand, we acknowledge limitations to our study. Especially the role of cycloplegic refraction in treatment planning is important when analyzing hyperopic treatment results. Although we focused on patients with minimal latent hyperopia, we did not analyze the influence of postoperative cycloplegic refraction. However, $\mathrm{Km}$ and refraction developed in a parallel manner that implies that regression is $\mathrm{Km}$ driven and not due to latent hyperopia.

\section{Conclusion}

In conclusion, after corneal laser refractive surgery, keratometric changes are followed by refractive changes and they occur up to 6 months after LASIK and for at least 6 months after PRK, and therefore, caution should be applied when retreatment is planned during the 1st year after surgery as 
hyperopic refractive regression can lead to suboptimal visual outcome. Keratometric and refractive stability is earlier achieved after LASIK, and therefore, retreatment may be independent of late regression.

\section{Disclosure}

The authors report no conflicts of interest in this work.

\section{References}

1. Spadea L, Sabetti L, D’Alessandri L, Balestrazzi E. Photorefractive keratectomy and LASIK for the correction of hyperopia: 2-year follow-up. J Refract Surg. 2006;22:131-136.

2. de Ortueta D, Arba Mosquera S. Topographic stability after hyperopic LASIK. J Refract Surg. 2010;26:547-554.

3. Jaycock PD, O'Brart DP, Rajan MS, Marshall J. 5-year follow-up of LASIK for hyperopia. Ophthalmology. 2005;112:191-199.

4. Kezirian GM, Moore CR, Stonecipher KG, et al; SurgiVision Consultants Inc WaveLight Investigator Group. Four-year postoperative results of the US ALLEGRETTO WAVE clinical trial for the treatment of hyperopia. J Refract Surg. 2008;24:431-438.

5. Kanellopoulos J, Asimellis G. Longitudinal postoperative lasik epithelial thickness profile changes in correlation with degree of myopia correction. J Refract Surg. 2014;30:166-171.

6. Katz T, Wagenfeld L, Galambos P, Darrelmann BG, Richard G, Linke SJ. LASIK versus photorefractive keratectomy for high myopic ( $>3$ diopter) astigmatism. J Refract Surg. 2013;29:824-831.

7. Katz T, Frings A, Richard G, Steinberg J, Druchkiv V, Linke SJ. Flapinduced astigmatism in eyes with sphere myopia correction: superior hinge using a rotating microkeratome versus nasal hinge using linear microkeratome. J Cataract Refract Surg. 2015;41:1160-1170.
8. Alió J, Galal A, Ayala MJ, Artola A. Hyperopic LASIK with Esiris/ Schwind technology. J Refract Surg. 2006;22:772-781.

9. Llovet F, Galal A, Benitez-del-Castillo JM, Ortega J, Martin C, Baviera J. One-year results of excimer laser in situ keratomileusis for hyperopia. J Cataract Refract Surg. 2009;35:1156-1165.

10. Waring GO 3rd, Fant B, Stevens G, et al. Laser in situ keratomileusis for spherical hyperopia and hyperopic astigmatism using the NIDEK EC-5000 excimer laser. $J$ Refract Surg. 2008;24:123-136.

11. Plaza-Puche AB, Yebana P, Arba-Mosquera S, Alió JL. Three-year follow-up of hyperopic LASIK using a 500-Hz excimer laser system. J Refract Surg. 2015;31:674-682.

12. Qazi MA, Roberts CJ, Mahmoud AM, Pepose JS. Topographic and biomechanical differences between hyperopic and myopic laser in situ keratomileusis. J Cataract Refract Surg. 2005;31:48-60.

13. Huang D, Tang M, Shekhar R. Mathematical model of corneal surface smoothing after laser refractive surgery. Am J Ophthalmol. 2003;135: 267-278.

14. Roberts C. Biomechanics of the cornea and wavefront-guided laser refractive surgery. J Refract Surg. 2002;18:589-592.

15. Mrochen M, Kaemmerer M, Mierdel P, Seiler T. Increased higher-order optical aberrations after laser refractive surgery: a problem of subclinical decentration. J Cataract Refract Surg. 2001;27:362-369.

16. Varley GA, Huang D, Rapuano CJ, et al; Ophthalmic Technology Assessment Committee Refractive Surgery Panel; Ameican Academy of Ophthalmology. LASIK for hyperopia, hyperopic astigmatism, and mixed astigmatism: a report by the American Academy of Ophthalmology. Ophthalmology. 2004;111:1604-1617.

17. Tabbara KF, El-Sheikh HF, Islam SM. Laser in situ keratomileusis for the correction of hyperopia from +0.50 to +11.50 diopters with the Keracor 117C laser. J Refract Surg. 2001;17:123-128.
Clinical Ophthalmology

\section{Publish your work in this journal}

Clinical Ophthalmology is an international, peer-reviewed journal covering all subspecialties within ophthalmology. Key topics include: Optometry; Visual science; Pharmacology and drug therapy in eye diseases; Basic Sciences; Primary and Secondary eye care; Patient Safety and Quality of Care Improvements. This journal is indexed on

\section{Dovepress}

PubMed Central and CAS, and is the official journal of The Society of Clinical Ophthalmology (SCO). The manuscript management system is completely online and includes a very quick and fair peer-review system, which is all easy to use. Visit http://www.dovepress.com/ testimonials.php to read real quotes from published authors. 